\title{
Disease progress and
}

\section{response to treatment as}

\section{predictors of survival,}

disability, cognitive

\section{impairment and depression in Parkinson's disease}

\section{Correspondence}

Professor Nick Holford MBChB FRACP Department Pharmacology \& Clinical Pharmacology, University of Auckland, 85 Park Rd, Private Bag 92019, Auckland, New Zealand.

Tel.: +64 (9) 9236730

Fax: +64 (9) 3737090

E-mail:n.holford@auckland.ac.nz

\section{Keywords}

disease progress, levodopa, Parkinson's disease, selegiline, time-dependent covariate, time to event

\section{Received}

21 January 2011

Accepted

25 January 2012

Accepted Article

Published Online

2 February 2012

\section{WHAT IS ALREADY KNOWN ABOUT} THIS SUBJECT

- The marked between variability in the rate of progression of Parkinson's disease severity assessed with a global functional score (Unified Parkinson' Disease Rating Scale, UPDRS) is recognized but its origin is uncertain and variously attributed to different subtypes of Parkinson's disease, life style, genetic variability and treatment. An increased risk of death in patients treated with selegiline has been reported but this is controversial.

\section{WHAT THIS STUDY ADDS}

-We used a hazard model approach to describe the time to clinical events (death, disability, depression and dementia). The time course of disease status changes was shown to be a key predictor of the risk of these events. Baseline motor subtype was not a predictor of outcome events. Selegiline was associated with an increased risk of death that was independent of its effect on disease status.

\section{AIM}

To describe the time to clinical events (death, disability, cognitive impairment and depression) in Parkinson's disease using the time course of disease status and treatment as explanatory variables.

\section{METHODS}

Disease status based on the Unified Parkinson's Disease Rating Scale (UPDRS) and the time to clinical outcome events were obtained from 800 patients who initially had early Parkinson's disease. Parametric hazard models were used to describe the time to the events of interest.

\section{RESULTS}

Time course of disease status (severity) was an important predictor of clinical outcome events. There was an increased hazard ratio for death $1.4(95 \% \mathrm{Cl} 1.31,149)$, disability $2.75(95 \% \mathrm{Cl} 2.30,3.28)$, cognitive impairment $4.35(95 \% \mathrm{Cl} 1.94,9.74)$, and depressive state $1.43(95 \% \mathrm{Cl}$ $1.26,1.63$ ) with each 10 unit increase of UPDRS. Age at study entry increased the hazard with hazard ratios of $49.1(95 \% \mathrm{Cl} 8.7,278)$ for death, $4.76(95 \% \mathrm{Cl} 1.10,20.6)$ for disability and $90.0(95 \% \mathrm{Cl} 63.3-128)$ for cognitive impairment at age 60 years. Selegiline treatment had independent effects as a predictor of death at 8 year follow-up with a hazard ratio of $2.54(95 \% \mathrm{Cl} 1.51,4.25)$ but had beneficial effects on disability with a hazard ratio of $0.363(95 \% \mathrm{Cl} 0.132,0.533)$ and depression with a hazard ratio of $0.372(95 \% \mathrm{Cl} 0.12,0.552)$.

\section{CONCLUSIONS}

Our findings show that the time course of disease status based on UPDRS is a much better predictor of future clinical events than any baseline disease characteristic. Continued selegiline treatment appears to increase the hazard of death. 


\section{Introduction}

Morbidity and mortality are not only related to progression of parkinsonian motor impairments but also to the emergence of other non-motor features such as depression, and cognitive impairment. Despite the biological plausibility of disease progression being correlated with clinical outcome in Parkinson's disease, many studies have explored only baseline or early clinical features and demographics as the potential risk factors for occurrence of clinical events in Parkinson's disease such as death, disability and dementia [1-3].

Because of the progressive nature of Parkinson's disease, the evolving disease status may predict future events. Reported predictors of events in Parkinson's disease are inconsistent as a result of patient selection, different study designs, analysis methods and outcome variables [1]. The relationship between anti-parkinsonian treatment and clinical events in Parkinson's disease is also unclear $[4,5]$ because typical analyses cannot account for the many changes in anti-parkinsonian medications that take place during the course of the disease.

We have used a fully parametric survival analysis model that can account for continuously time-varying factors such as disease status and its response to treatment. Parametric models offer robustness for heavily intervalcensored data, as in our case, and more precise inferences if the assumption of the distribution of event times is valid [6-8]. A review of the concepts of population approach methodology applied to clinical pharmacology is recommended for the reader who may find some of our methods and interpretation of results unfamiliar [9]. The reader is also referred to an overview of the use of disease progress modelling in Parkinson's disease [10].

Survival in Parkinson's disease was described for the DATATOP cohort in 1998 by the Parkinson Study Group (10 years follow-up) [11] and in 2005 by Marras et al. (13 years follow-up) [12]. Marras et al. used a semi-parametric modelling method and found that better survival was related to levodopa responsiveness and worse survival was related to disease severity in early stages of the disease. Our parametric method has been applied to survival time as well as the time to reach a certain level of disability, impaired cognitive function and mood change. We have investigated the hypothesis that clinical events in Parkinson's disease are better predicted by the time course of disease progress and anti-parkinsonian treatments rather than baseline variables alone. The rationale for studying the influence of continuously predicted, instead of intermittently observed, disease status is twofold: the time course of the disease is more accurately captured and the influence of treatment effects on clinical events can be distinguished; that is, a treatment may influence clinical events indirectly by affecting disease progression, via symptomatic or disease-modifying effects, or directly through adverse effects.

\section{Methods}

\section{Event times data}

Data were provided by the Parkinson Study Group. Event times for death, disability, cognitive impairment and depression were obtained from the DATATOP cohort. Various categories (not mutually exclusive) describing atypical features or likelihood of not having PD were included in the data set. These categories (number of patients) were: PD not confirmed by autopsy (5), imaging study suggests other cause (15), less than $40 \%$ confident of PD diagnosis (33), little or no response to levodopa (49), PD not among the three most likely diagnoses (19), atypical PD features or disease course (11), postural hypotension (52), oculomotor, eyelid, other neurologic features (54), dementia less than or equal to 5 years after PD onset (22).

Details of the DATATOP study trial design and patient characteristics have been published $[13,14]$. Briefly, we distinguish the DATATOP study which enrolled 800 patients into a randomized trial of selegiline, tocopherol and placebo which lasted about 2 years. Five of these patients dropped out early in the clinical trial and one of these did not have a record of the dropout date. The DATATOP cohort included 795 patients who were followed during the DATATOP study and subsequently for nearly 8 years. Treatment information, patient demographics and clinical assessments were obtained from the DATATOP cohort. The average follow-up time was 4.97 years with a maximum of 7.73 years. Follow-up clinical assessments were made approximately every 3-6 months and included UPDRS [15], mini mental state examination (MMSE) [16] and Hamilton depression rating scale (HAM-D) [17] assessments. After the end of the study at 7.73 years, there was no further record of clinical state or treatment during the follow-up period of the DATATOP cohort.

We defined non-death events using cut off values of activities of daily living (ADL) scores for disability, MMSE scores for cognitive impairment and HAM-D for mood state. ADL scores were derived from the UPDRS subpart II. The cut off values for event definitions were selected based on the minimal clinically important changes studied in patients with Parkinson's disease $[18,19]$ and sufficient numbers of events for meaningful analysis. It is recognized that these minimal clinical important changes are neither dementia nor major depressive illness. However, there is clinical interest in describing early cognitive and emotional changes that may affect quality of life as well as herald dementia and major psychiatric disorders. A disability event (ADL15) was defined by an ADL score of 15 or greater during the 'on' state (i.e., presence of motor response to treatments). We defined disability events using the threshold of ADL score instead of total UPDRS (parts I-III) because the ADL score is more specific to patients' daily function and is not confounded with more specific motor impairments that may or may not cause disability. A 
cognitive impairment event (MMSE24) was defined by an MMSE score of 24 or less. Finally, a HAM-D score of 10 or more defined a depression event (HAMD10).

An event time was defined as the time when an event or endpoint occurred after a patient entered the study, and was considered censored if it had not been observed for a patient at the end of 8 years follow-up, or if the patient had dropped out from the study before 8 years. For non-death events, we defined event time as the time when the patient first met or exceeded the threshold score for the event at any scheduled visit. The actual time of a non-death event was not known except that it occurred at some time between the previous clinic visit and the visit at which the event threshold had been reached. Death events were considered to have occurred over an interval of 1 day. All event times were therefore treated as interval censored data. That is, the event was assumed to occur at an unknown time during a known interval.

Survival times were available from a 14 year follow-up of vital status for the cohort, previously reported by Marras et al. [12]. A subset of death event times was created covering the period during which regular assessments of Parkinson's disease status were obtained and treatment recorded. Deaths up to 6 months after the last visit were included otherwise censoring for deaths occurred at the time of the last visit. This death event subset is referred to as the EOT data set.

We did not exclude patients who might have had a diagnosis of something other than idiopathic Parkinson's disease (i.e., level of confidence $<60 \%$ for idiopathic Parkinson's disease) because the number of these cases was small (53 out of 800 ) and previous survival analysis showed that these patients had no significant impact on the results [12].

\section{Parametric time to event models}

The time to an event, $T$, a random variable occurs at time $t$ after study entry. Using a parametric time to event model, the probability density distribution of $T$ can be explicitly defined through the hazard function [6]. The hazard function quantifies the instantaneous rate of experiencing an event and is useful in time to event modelling because the influence of explanatory variables, especially those that vary continuously with time (such as disease status), can be directly included. The simplest parametric hazard model assumes a constant hazard rate at any time after study entry which leads to an exponential distribution of $T$. For non-constant hazard, the Gompertz \& Weibull distributions of $T$ are commonly used to describe the time-varying shape of the underlying hazard function [6]. We investigated three baseline hazard functions $h_{0}(t)$ as shown in equation (1), where $\beta_{0}$ is a parameter common to all three functions and $\beta_{\text {time }}$ is a parameter that relates $t$ to $h_{0}(t)$. The expression of the Weibull distribution hazard function shown in equation (1) makes clear its relationship to the other two distributions.
Exponential: $h_{0}(t)=\beta_{0}$

$$
\begin{aligned}
& \text { Gompertz: } \quad h_{0}(t)=\beta_{0} \times \exp \left(\beta_{\text {time }} \times t\right) \\
& \text { Weibull: } h_{0}(t)=\beta_{0} \times \exp \left(\beta_{\text {time }} \times \ln (t)\right)
\end{aligned}
$$

\section{Explanatory variables}

Potential variables that may explain the change in hazard function (i.e. covariates) are of two types, time varying and constant (e.g., baseline clinical features). Time varying covariates include predicted disease status and antiparkinsonian treatment. Continuous time varying disease status was computed using disease progress models previously described for total UPDRS [20], UPDRS subscales (i.e. tremor, rigidity, bradykinesia, postural instability gait disorder [PIGD] and $A D L$ ) and non-motor features (i.e. MMSE, and HAM-D) [21]. Anti-parkinsonian treatments (e.g. on or off selegiline) are time varying covariates that remain constant over defined intervals. Fixed covariates consisted of patients' characteristic at study entry: motor subtypes (i.e. tremor-dominant, PIGD-dominant and indeterminate subtypes), gender, age and smoking status. Tremordominant and PIGD-dominant subtypes were defined as suggested by Jankovic et al. [22].

The parameter estimates of the hazard functions are more readily understood by converting the Gompertz parameter to a half-life by dividing the estimate into $\ln (2)$ (and other hazard parameters to hazard ratios by exponentiation of the estimate. 95\% confidence intervals were calculated from the original parameter estimates and asymptotic standard errors. The limits of these intervals were then transformed into half-lives or hazard ratios.

\section{Joint models of disease progress and time to event}

Disease progress models were previously fitted to disease status markers measured in the DATATOP cohort using a nonlinear mixed effects approach [20, 21]. Individual disease progress parameters (IPP) were obtained from empirical Bayes estimates based on these models. The IPP were combined with event time data for joint computation of the predicted time course of disease status and the probability of events.

The hazard function $h(t)$ is now dependent on a set of $p$ covariates which may be time-dependent $X(t)=x_{1}(t), x_{2}(t)$, $\left.x_{3}(t) \ldots, x_{p}(t)\right)$ with size of effect measured by a set of coefficients $\left(\beta=\beta_{1}, \beta_{2}, \beta_{3}, \ldots, \beta_{p}\right)$ and $h_{0}(t)$ is the baseline hazard [equation (2)]. Disease status, including the effects of treatment, is an example of a time varying covariate.

$$
h(t)=h_{0}(t) \times \exp \left(\sum_{j=1}^{p} \beta_{j} \times x_{j}(t)\right)
$$

The hazard ratio reflecting the influence of a covariate can be obtained from $\exp (\beta \cdot x(t))$, From the hazard function, the probability that an event time is greater than or equal 
to time $t$ (i.e. the survivor function $\mathrm{S}(t)$ ) can be determined by integrating the hazard function [equation (3)].

$$
\mathrm{S}(t)=\operatorname{Pr}(T \geq t)=\exp \left(-\int_{0}^{t} h(u)\right) d u
$$

All event times were treated as interval censored data that assumed the exact $T$ was unknown and occurred between the previous time when the event had not occurred $\left(t_{\text {pre }}\right)$ and the time when the event was first detected as having occurred $\left(t_{\text {post }}\right)$. The likelihood for interval censored $T$ was computed as $S\left(t_{\text {pre }}\right)-S\left(t_{\text {post }}\right)$ [6]. Death events were assumed to have a censoring interval of 1 day. Other event intervals were determined by the time of the visit before the event and the time of the visit after the event.

No random effect models were used for the estimated parameters of the time to event models. However, fixed and random differences in between subject variability were included through the use of individual treatment patterns and empirical Bayes estimates of disease progress parameters.

\section{Dropout model}

A dropout model was also developed for the purpose of simulating censored events. The dropout model was a parametric time to event model tested for covariate effects as described in the previous section. Time to dropout was defined as the time of the last visit before the nominal end of the trial at 8 years or died before 8 years. Thus all patients had this kind of event because no patient was followed with full treatment records for more than 7.73 years. There were no censored events for the dropout model because everyone had the event of not reaching the 8 year nominal end point. Separate hazard functions were used for death and for non-death causes of not reaching 8 years.

\section{Parameter estimation}

The Laplacian estimation method in NONMEM software (version Vl, release 1.3) [23] was used to obtain maximum likelihood estimates of time to event parameters.

\section{Model selection and evaluation}

Covariate inclusion/exclusion was determined by a decrease/increase in NONMEM objective function value (OFV) of at least 7 units for one additional parameter, which is approximately equivalent to a $\chi^{2}$-distributed critical value for $P<0.01$ with 1 degree of freedom [9]. When comparing non-nested models (e.g. disease status as UPDRS or its subscales), the statistical significance criterion for model selection we used was a difference of 10 units in OFV. Parametric models without covariates were first fitted to the time to event data. The influence of individual covariate effects was evaluated by including each covariate separately in the hazard function. Combinations of the covariates were then examined for interactions. During the model-building process, influential covariates were retained in the final models after stepwise addition and deletion. The imprecision of model parameters was determined by asymptotic standard errors generated by NONMEM.

Residual diagnostics were not particularly useful for models with time-dependent covariates because there were many different combinations of covariate values for any one individual [6], and therefore they were not employed in this analysis. Instead, a visual predictive check (VPC) was used to evaluate the model appropriateness [24-26]. The procedure involved first using NONMEM to simulate distribution of event times for the same 795 patients from the DATATOP cohort with their dosing information and predicted disease progress. Then, using the Kaplan-Meier method in the $R$ software [27], nonparametric survivor function estimates were computed for these simulated event times. After repeating these two steps 100 times the $5^{\text {th }}, 50^{\text {th }}$ and $95^{\text {th }}$ percentiles of the Kaplan-Meier estimates of the survivor function were calculated and overlaid with a Kaplan-Meier plot of the observed event times. In order to simulate realistic censoring of events, the dropout model was included in the simulation for non-death events.

\section{Results}

The analysis data set included DATATOP subjects who had at least two visits. The median age at entry was 62.7 years with $95 \%$ of patients between 39.7 and 77.2 years. Table 1 shows the median event times and the number of evaluable events for disability (ADL15, an ADL score of 15 on the UPDRS part II), cognitive impairment (MMSE24, an MMSE score of 24 or less) and depression (HAMD10, a score of 10 or greater on the HAM-D).

The distribution of event times suggested a nonconstant hazard for each type of event (Figure 1). The event hazard changed exponentially with time as described by a Gompertz distribution for death, ADL15 and HAMD10. There was no association of MMSE24 with time that was not accounted for by changes in disease severity.

Half-lives for the Gompertz distribution and hazard ratios with their corresponding 95\% confidence intervals

\section{Table 1}

Summary of event times in the analysis data set

\begin{tabular}{|llll|}
\hline & $\begin{array}{l}\text { Total } \\
\text { evaluable } \\
\text { observations }\end{array}$ & $\begin{array}{l}\text { Total } \\
\text { non-censored } \\
\text { events (\%) }\end{array}$ & $\begin{array}{l}\text { Median event } \\
\text { time, years } \\
\text { (range) }\end{array}$ \\
\hline $\begin{array}{l}\text { Deaths at EOT } \\
\text { Disability (ADL15) }\end{array}$ & 799 & $98(12 \%)$ & $5.03(0.95-7.68)$ \\
$\begin{array}{l}\text { Cognitive impairment } \\
\text { (MMSE24) }\end{array}$ & 789 & $364(47 \%)$ & $1.30(0.13-7.52)$ \\
\hline $\begin{array}{l}\text { Depression (HAMD10) } \\
\text { (Ments }\end{array}$ & 771 & $89(11 \%)$ & $2.84(0.13-7.34)$ \\
\hline
\end{tabular}



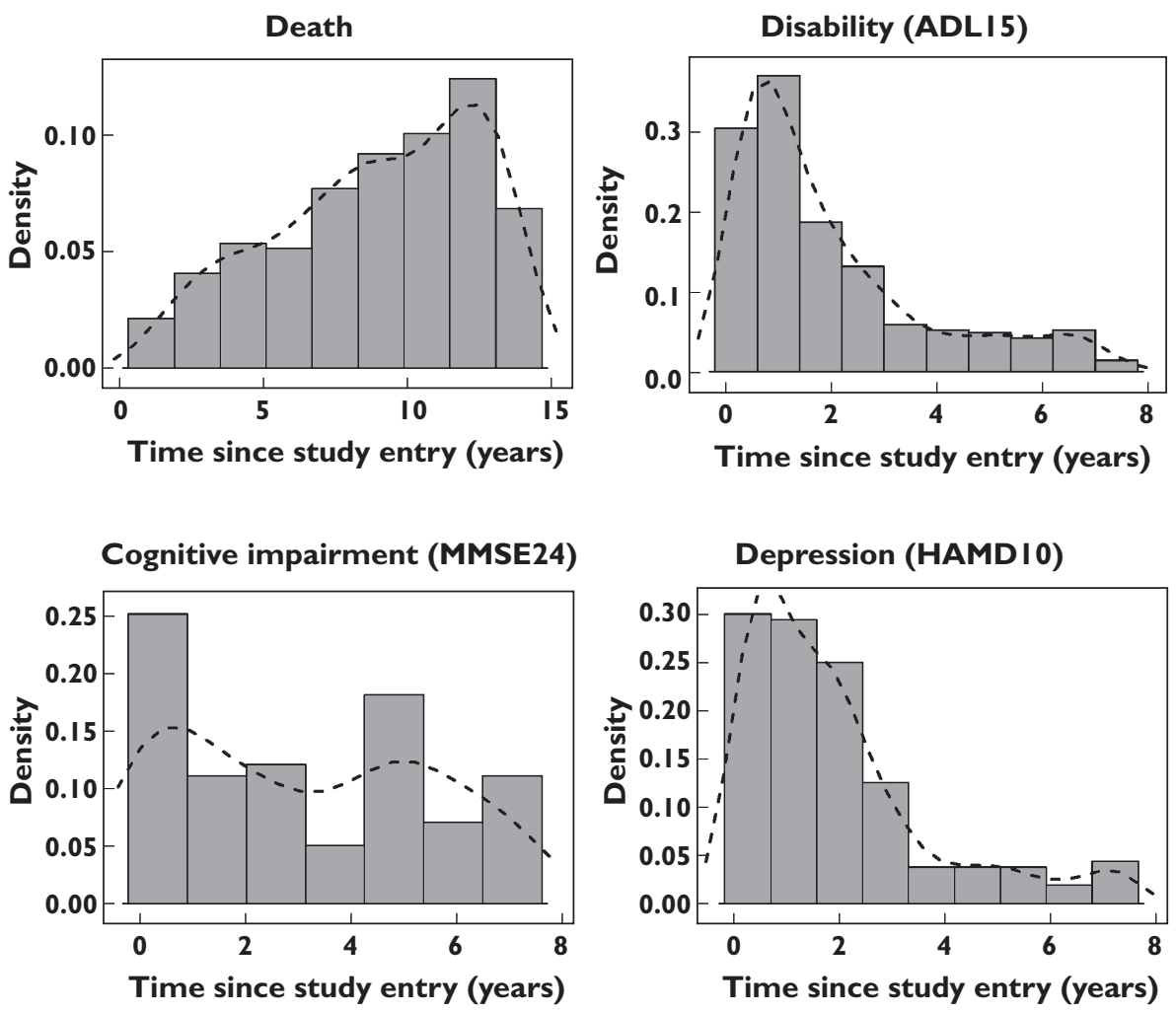

Figure 1

Distribution of event times (in density scale) with a kernel density smooth (dashed lines)

\section{Table 2}

Half lifes of Gompertz hazard increase and hazard ratios for death events

\begin{tabular}{|lccc|}
\hline & $\begin{array}{l}\text { Death EOT } \\
\text { Estimate }\end{array}$ & $\mathbf{2 . 5 \% i l e}$ & $\mathbf{9 7 . 5 \% i l e ~}$ \\
\hline Half-life (Gompertz) & 5.46 & 26.0 & 3.05 \\
\hline HR of $\mathbf{4 0}$ years at entry & 13.4 & 4.22 & 42.6 \\
\hline HR of $\mathbf{6 0}$ years at entry & 49.1 & 8.7 & 278 \\
\hline Ratio $\mathbf{6 0}$ years : $\mathbf{4 0}$ years & 3.66 & 2.06 & 6.52 \\
\hline HR of selegiline & 2.54 & 1.51 & 4.25 \\
\hline HR of levodopa & - & - & - \\
\hline HR of $\mathbf{1 0}$ unit status increase & 1.40 & 1.31 & 1.49 \\
\hline
\end{tabular}

are shown in Tables 2 and 3. Although dominant motor subtype defined at baseline was not a significant covariate for any of the events, the tremor-dominant group consistently had a smaller hazard ratio for all events. For example, the hazard ratio for death at EOT was $1.08(95 \% \mathrm{Cl} 0.32$, 2.58) for tremor and $1.28(95 \% \mathrm{Cl} 0.4,1.52)$ for PIGD disease status.

\section{Predictors of survival}

Because of colinearity between time-dependent variables, the influence of some covariates changed when others were present in the models. For instance, the influence of selegiline treatment on hazard was not significant when tested alone nor when combined with time and age at study entry. However, when disease progress was added to the model, selegiline treatment was associated with a significantly increased hazard of death. The increased hazard was only associated with those times during the 8 years that selegiline was used. At the same time there was a beneficial effect on the hazard of death through its symptomatic and disease modifying influences on disease status as described by the UPDRS.

The hazard of death during the study period increased with time, age at study entry and worsening UPDRS. Selegiline treatment, independent of its effect on disease severity, was associated with an increased risk of death with a hazard ratio of $2.54(95 \% \mathrm{Cl} 1.51,4.25)$ with a daily dose of $10 \mathrm{mg}$. The time course of the hazard is shown in Figure 2 for three hypothetical populations assigned to placebo, levodopa alone or levodopa plus selegiline for 8 years. The levodopa treated population has a lower hazard compared with placebo at all times. This is explained by the improvement in UPDRS and not by levodopa treatment itself. Combination with selegiline increases the hazard of death compared with placebo and levodopa alone. The increased hazard lasts for about 1.5 years before dropping below the placebo, then at about 5 years the additive 
Table 3

Half-lives of Gompertz hazard increase and hazard ratios for disability (ADL15), cognitive impairment (MMSE24) and depression (HAMD10)

\begin{tabular}{|c|c|c|c|c|c|c|c|c|c|}
\hline Explanatory factor & $\begin{array}{l}\text { ADL15 } \\
\text { Estimate }\end{array}$ & $2.5 \%$ ile & $97.5 \%$ ile & $\begin{array}{l}\text { MMSE24 } \\
\text { Estimate }\end{array}$ & $2.5 \%$ ile & $97.5 \%$ ile & $\begin{array}{l}\text { HAMD10 } \\
\text { Estimate }\end{array}$ & $2.5 \%$ ile & $97.5 \%$ ile \\
\hline Half-life (Gompertz) & 2.77 & 4.56 & 1.99 & - & - & - & 1.80 & 2.55 & 1.39 \\
\hline HR of 40 years at entry & 2.83 & 1.06 & 7.53 & 20.1 & 15.9 & 25.4 & - & - & - \\
\hline HR of 60 years at entry & 4.76 & 1.10 & 20.6 & 90.0 & 63.3 & 128 & - & - & - \\
\hline Ratio 60 years : 40 years & 1.68 & 1.03 & 2.74 & 4.48 & 3.98 & 5.04 & - & - & - \\
\hline HR of selegiline & 0.36 & 0.13 & 0.53 & - & - & - & 0.37 & 0.12 & 0.55 \\
\hline HR of levodopa & - & - & - & - & - & - & - & - & - \\
\hline HR of 10 unit status increase & 2.75 & 2.30 & 3.28 & 4.35 & 1.94 & 9.74 & 1.43 & 1.26 & 1.63 \\
\hline
\end{tabular}

HR, hazard ratio.

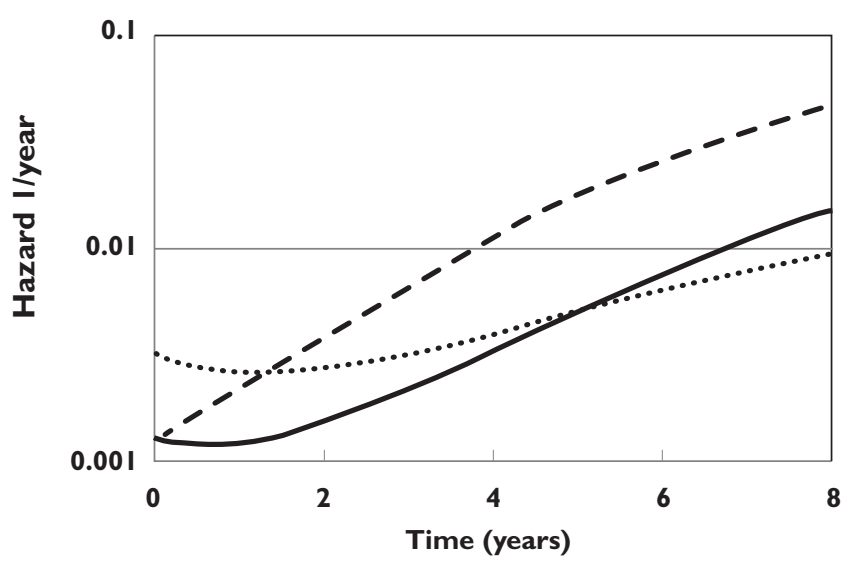

\section{Figure 2}

Time course of hazard of death (EOT) for placebo (dashed line), levodopa $300 \mathrm{mg} \mathrm{day}^{-1}$ (solid line) and levodopa $300 \mathrm{mg} \mathrm{day}^{-1}$ plus selegiline $10 \mathrm{mg} \mathrm{day}^{-1}$ (dotted line)

symptomatic and synergistic disease modifying effects of selegiline [20] lead to a hazard lower than treatment with levodopa alone. The model predictions describe the probability of survival and are in good agreement with the Kaplan-Meier plot for the observed survival (Figure 3).

Each of the nine categories describing atypical features of PD or absence of PD were investigated as potential explanatory factors for the hazard of death. Most categories were associated with a significantly increased hazard of death with the exception of 'imaging study suggests other causes' and 'postural hypotension'. The significant association of selegiline treatment with increased hazard of death remained for all categories.

\section{Predictors of time to event for non-death events}

ADL15 The hazard of disability decreased with time, but increased with age at entry and UPDRS score. Selegiline treatment minimally reduced ADL disease severity (interpreted as a symptomatic effect) [21] but was associated with a lower hazard of reaching the ADL15 endpoint as shown by a hazard ratio of $0.363(95 \% \mathrm{Cl} 0.132,0.533)$, a disease modifying effect. On the other hand, levodopa reduced $A D L$ disease severity to a much greater extent than seligiline (symptomatic effect) [21] but did not reduce the hazard of disability that was independent of its symptomatic effect on disease status.

MMSE24 The hazard ratio of MMSE24 events increased with age at entry and with the worsening of the PIGD sub-scale of UPDRS. There was no apparent increase in hazard with time. Neither levodopa nor selegiline treatment had a significant effect on the hazard.

HAMD10 The hazard decreased with time and with the worsening of UPDRS score. Selegiline treatment decreased the hazard of reaching the HAMD10 endpoint as shown by a hazard ratio of $0.372(95 \% \mathrm{Cl} 0.12,0.552)$. There was no significant association with age at entry or being on levodopa treatment independent of its effect on disease status.

Model predictions described the observed events of ADL15, MMSE24 and HAMD10 with generally good agreement with the observed Kaplan-Meier plots (Figure 3).

\section{Dropout model}

The hazard of dropout (i.e. failing to reach 8 year follow-up) due to causes other than death was described by the sum of two Gompertz distributions based on time and a Weibull distribution based on In(time) (see supplemental results, Table A3). The time course of the hazard is shown in Figure 4 for three hypothetical populations assigned to placebo or levodopa alone or levodopa plus selegiline for 8 years. The baseline hazard of the first Gompertz distributions $\left(0.0764\right.$ year $\left.^{-1}\right)$ was much larger than the second $\left(0.0524 \times 10^{-9}\right.$ year $\left.^{-1}\right)$. The first distribution decreased with time with a half-life of $1.66(95 \% \mathrm{Cl} 1.16,2.91)$ years while the second increased with a half-life of $2.29(95 \% \mathrm{Cl} 2.06$, 2.57) years. The Weibull distribution component of the hazard increased with time and largely explained the loss of subjects as the follow-up period approached its end. 

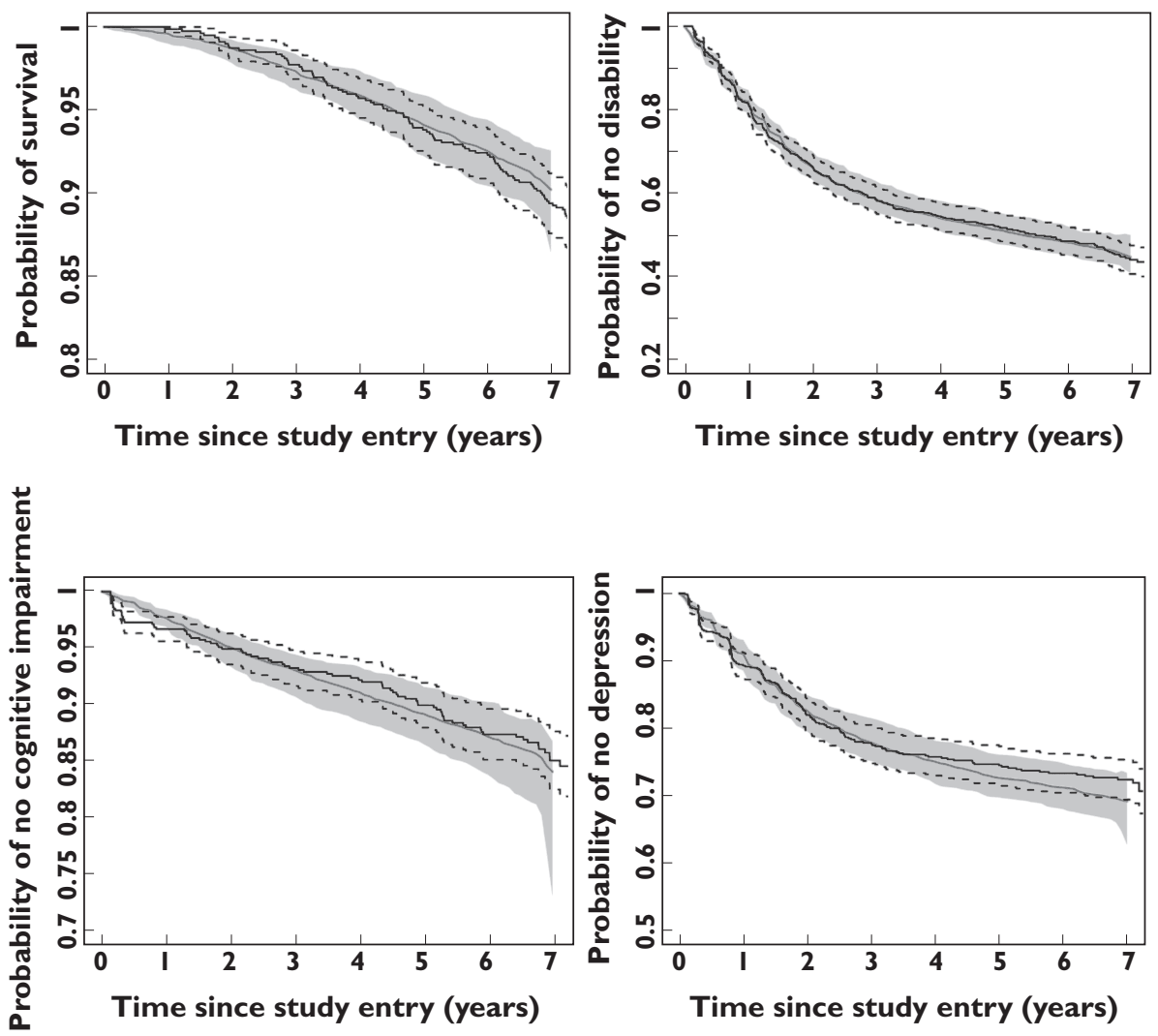

\section{Figure 3}

Kaplan-Meier estimates of observed time to event data (solid lines) and associated $90 \%$ confidence intervals (dotted lines), overlaid with parametric model predictions of the base models (dashed lines) and their $90 \%$ confidence intervals (gray bands)

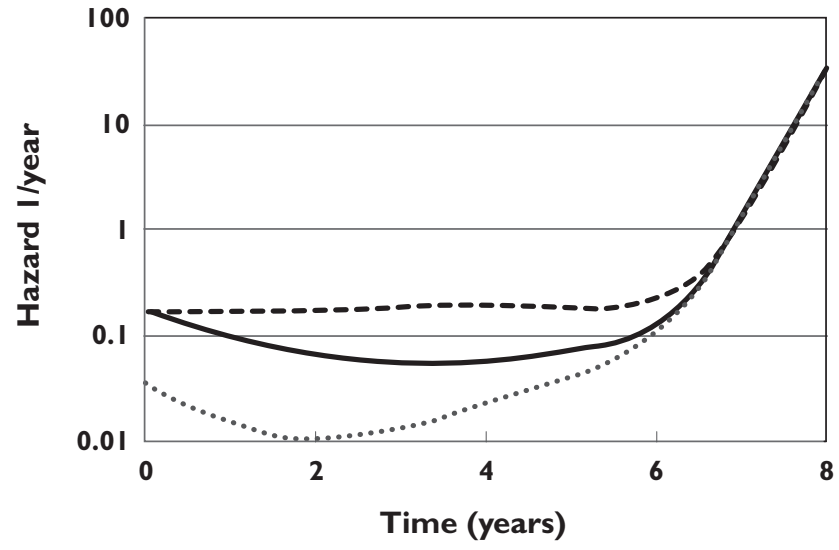

\section{Figure 4}

The time course of hazard of dropout for placebo (dashed line), levodopa $300 \mathrm{mg} \mathrm{day}^{-1}$ (solid line) and levodopa $300 \mathrm{mg} \mathrm{day}^{-1}$ plus selegiline $10 \mathrm{mg} \mathrm{day}^{-1}$ (dotted line)

The explanatory factors for selegiline and UPDRS hazard were applied to the first Gompertz baseline distribution because almost all of the baseline hazard was associated with this component. The hazard of dropout was predicted to be almost constant for untreated patients. The hazard ratio of dropout associated with selegiline treatment was $0.202(95 \% \mathrm{Cl} 0.133,0.305)$ which indicated selegiline reduced the risk of dropping out. Worsening of UPDRS score had a higher hazard of dropout with a hazard ratio of $1.48(95 \% \mathrm{Cl} 1.30,1.68)$.

\section{Discussion}

We have explored factors predicting the time course of hazard for death, ADL15 (disability), MMSE24 (cognitive impairment) and HAMD10 (depression) by linking disease time course to the times of these clinical events. Our analysis indicated that the hazards of clinical events were not constant over time but were influenced strongly by the time course of disease status. The importance of considering the time course of treatment induced changes in biomarkers has been demonstrated by differences in risk of renal impairment related to time varying changes in biomarkers of diabetes [28]. The time course of UPDRS status is a much better predictor of future clinical events than any baseline disease characteristic. Graham et al. showed that the individual slopes and intercepts from a linear model for partial MMSE helped improve the fit for a time to dropout 
event model in patients with cognitive impairment [29]. However, they did not consider the time course of the partial MMSE as an explanatory factor for dropout. On the other hand using the time course of CD4 count progression in a survival model was able to act as a surrogate for treatment effects as a predictor of survival in patients with AIDS [30]. Similar results were obtained to predict survival from the time course of lymphocytes in chronic lymphatic leukemia [31].

Because there is evidence that the motor features of Parkinson's disease (tremor, rigidity, bradykinesia and PIGD) progress at different rates [32], the effect of each of these features on clinical events in Parkinson's disease may be different. We found that tremor did not predict clinical events unlike the other Parkinson's disease features (Table A1). Despite reports of baseline PIGDdominant subtype as an important predictor for worsening disability and dementia [22, 32,33], we found this baseline subtype classification was neither a significant factor influencing hazard for these events nor for the hazard of death or depression. Tremor was only a weak predictor of disability and did not influence the other endpoints we examined.

We found that selegiline treatment increased the hazard for mortality at EOT, which was independent of its beneficial effects on mortality by disease progress benefits. A semi-parametric survival analysis of the DATATOP cohort at 10 year follow-up did not detect a difference in death rates between the original randomized groups of selegiline and no-selegiline (i.e. tocopherol or placebo) [11]. The main drawback of this 10 year analysis was that death rates were associated only with the original intention to treat randomization and thus did not account for actual use of selegiline. This is a major weakness of the intention to treat approach because it ignores what was actually known to happen. We tested selegiline treatment as an intention to treat variable i.e. using the original selegiline randomization and ignoring the known changes in selegiline treatment. The effect of selegiline, under the intention to treat assumption, was not statistically significant. A meta-analysis of five randomized trials (not including DATATOP) of selegiline or levodopa/placebo in patients with early Parkinson's disease with a mean follow-up of 4 years also did not find an increase in mortality in the selegiline treated group [5]. However, it is difficult to draw meaningful conclusions from this study because of the low overall death rate over 4 years (4\%).

We found that inclusion of selegiline treatment time course alone was not a significant predictor of mortality ( $P$ $>0.01$, Table A1). However, when selegiline treatment was included with the time course of disease progress, the increased hazard became apparent $(P<0.001)$. This is because selegiline lowers the hazard through its beneficial effects on disease status $[20,21]$ so that the adverse effect of selegiline is concealed if the hazard is not adjusted for disease status effects.
In an open-label prospective randomized study of 520 patients with early Parkinson's disease, the Parkinson's Disease Research Group of the United Kingdom (PDRG-UK) found a $57 \%$ higher risk of mortality in patients taking a combination of selegiline and levodopa compared with levodopa alone (adjusted hazard ratio $1.57,95 \% \mathrm{Cl} 1.07$, 2.31) [4]. Our estimated hazard of death associated with selegiline is higher, which may be because we have included the time-varying effects of selegiline on hazard to account for on and off treatment periods during the trial. A 2 year follow-up from the PDRG-UK, after all patients had stopped selegiline, reported a similar death rate between the two treatment groups [34], which further substantiated an association of selegiline with increased mortality. Following the PDRG-UK study, an observational crosssectional study of 12621 patients in UK found a nonsignificant but also increased risk of death (11\%) in patients taking either selegiline alone or in combination with levodopa [35].

Since the publication of the PDRG-UK study results, several clinical studies have attempted to investigate the possible cardiotoxic effect of selegiline in patients with Parkinson's disease. These studies noted that patients taking selegiline long term had a higher risk of orthostatic hypotension [36-39]. However, no studies have yet shown a causal relationship between selegiline and cardiotoxicity that may explain the increased death rate. Cause of death was only determined for 29 patients in the DATATOP cohort and the most common cause was pneumonia. Only three patients died of myocardial or cerebral infarction in the selegiline group compared with none in the other treatment groups [11]. Therefore, we could not determine the association of cardiovascular toxicity with selegiline treatment.

There are several limitations in our analysis. Firstly, our analysis is retrospective, exploratory and descriptive in nature, and therefore, causal inferences are only suggestive. This is similar to other reports that have attempted to investigate this issue. However, the interconnected relationship of disease status and clinical events provides insights about the underlying mechanism and the effects of treatment. We believe that our use of the rate of progression of disease and its modification by treatment provides an essential clue in understanding why other retrospective studies of the same data may have been unable to find an association of selegiline with increased risk of death. The confirmation of increased risk of selegiline use would require a long term randomized prospective trial.

Seven of nine categories describing atypical or less than certain diagnosis of PD were associated independently with an increased hazard of death but this did not account for the hazard associated with selegiline treatment. Therefore, the association of selegiline treatment with an increased hazard of death does not appear to be an artifact due to misdiagnosis. We report the hazard associated with selegiline based on all patients because the 
decision to use selegiline was typically made before these atypical features were evident.

Secondly, the criteria for defining disability, cognitive impairment and depression are not equivalent to standard clinical diagnostic criteria. We were unable to apply stricter cut off values to define psychiatric events and yet retain sufficient numbers of events for meaningful investigation. Because the clinical diagnosis of dementia was not available in the DATATOP database, we evaluated a cognitive impairment event (MMSE $\leq 24)$ as a surrogate for mild dementia [40]. One prospective population-based study assessed the incidence of dementia in patients with Parkinson's disease and found that a baseline MMSE score of less than 29 was a significant risk factor for dementia [41]. Similarly, we did not have a clinical diagnosis of depression available for the cohort. A HAM-D score of 10 or more was used instead to determine a depression event. This cut off score had been used to screen for depression in patients with Parkinson's disease [18] and studies suggested that patients might have depressive symptoms prior to the onset of Parkinson's disease [42].

In conclusion, we have shown the value of timedependent disease progress as a predictor of clinical events in Parkinson's disease. We have taken a parametric survival analysis approach and found a significant association between continued selegiline treatment and mortality, a controversial finding that was first reported by the PDRG-UK study [4]. By linking the disease progress models to time to event models, we were able to differentiate the beneficial effects (i.e. symptomatic and/or diseasemodifying) and the adverse effects of anti-parkinsonian medications on the progression of Parkinson's disease. Our analytical approach can accommodate the varied individual patterns of dosing regimens and disease progression, and can be updated or validated with an external data set with different designs. Finally, the models may be useful in determining prognosis based on early disease progression observations (e.g. 2 years after initial diagnosis) [43].

Our analysis is in agreement with other studies that selegiline treatment is associated with increased risk of death in Parkinson's disease patients. This increased risk is mitigated in part by the beneficial effects of selegiline in improving disease status which in turn reduces the risk. However, if selegiline is used continuously we predict an overall increase in mortality compared with not using selegiline especially in the early years before the disease modifying effects of selegiline are prominent. The intermittent use of selegiline in the DATATOP cohort reduces the cumulative hazard and would explain why earlier analyses could not find evidence for increased deaths associated with selegiline.

\section{Competing Interests}

N. Holford has received consulting fees from Novartis. Novartis produces anti-Parkinsonian medication. There are no other competing interests to declare.

This work was supported by a grant from the Michael J. Fox Foundation. We are grateful to the Parkinson Study Group for providing access to the DATATOP cohort and to $\operatorname{Dr} C$. Marras for providing additional information about patient survival. We wish to acknowledge the continuing support and encouragement of the Parkinson Study Group and Dr Arthur Watts, University of Rochester, for help in providing the data for the DATATOP cohort.

\section{Appendix}

The following tables are supplemental results for the time to event models in the main article.

Table A1 shows the model selection sequence for each time to event model based on statistical significance (e.g. change in objective function values). There was no evidence to support the use of In(time) as an explanatory factor (Weibull distribution) as demonstrated by the small change in objective function value of 3 units when the Weibull distribution using In(time) was added to the Gompertz distribution using time. For purposes of comparison with other covariates, the objective function value for models of time and age at entry are shown. 


\section{Table A1}

Model selection. The objective function change associated with using different baseline hazard functions and other explanatory variables is shown

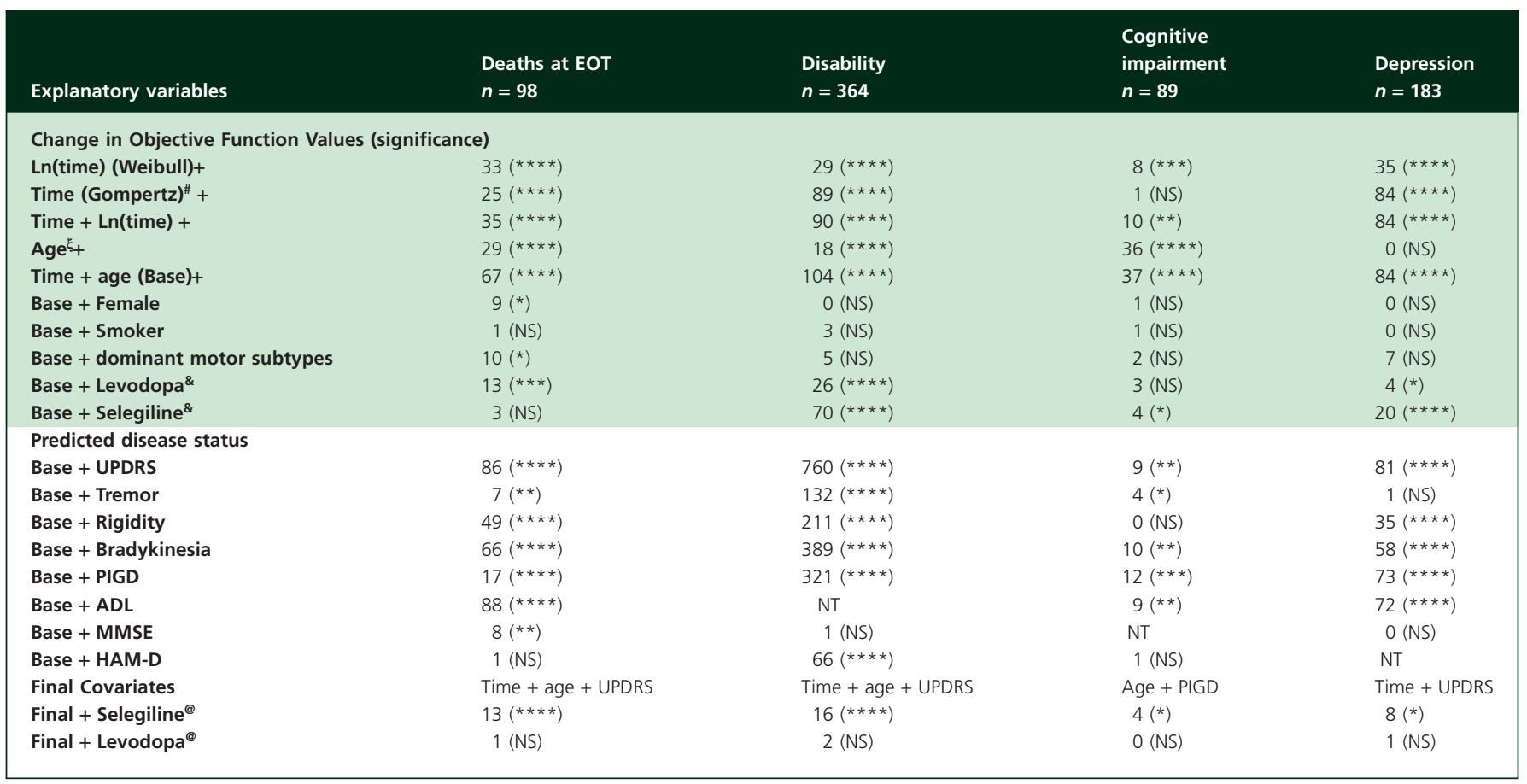

EOT, end of trial; UPDRS, United Parkinson's Disease Rating Scale; PIGD, postural instability gait disorder score; ADL, activities of daily living score; MMSE, minimum mental state examination score; HAM-D, Hamilton depression score; NT, not tested for inclusion.

"Time since study entry (Gompertz hazard); ${ }^{\sharp}$ Age at study entry; ${ }^{*}$ Reference model is a constant-hazard model; ${ }^{\oplus}$ Reference model is the Final Covariates model; ${ }^{8}$ On treatment is 1 and off-treatment is 0 .

$* * * *$ value $<=0.0001 ; * * * P$ value $<=0.001 ; * * P$ value $<=0.01 ;{ }^{*} P$ value $<=0.05$. NS $=$ not significant.

\section{Table A2}

Summary of time to event parameters for the final models RSE (in parentheses) is the relative standard error calculated as a percentage of the parameter point estimate

\begin{tabular}{|c|c|c|c|c|}
\hline \multirow[b]{2}{*}{ Parameters } & \multicolumn{4}{|c|}{ Parameter estimates (RSE \%) } \\
\hline & Deaths at EOT $n=98$ & $\begin{array}{l}\text { Disability } \\
n=364\end{array}$ & $\begin{array}{l}\text { Cognitive } \\
\text { impairment } \\
n=89\end{array}$ & $\begin{array}{l}\text { Depression } \\
n=183\end{array}$ \\
\hline Baseline hazard & $1.28 \times 10^{-5}$ & $2.45 \times 10^{-3}$ & $1.39 \times 10^{-4}$ & $5.79 \times 10^{-2}$ \\
\hline$\beta_{0}$, u year ${ }^{-1}$ & (18) & (58) & (10) & (27) \\
\hline $\begin{array}{l}\text { Time } \\
\beta_{\text {time, }} \text { year }^{-1}\end{array}$ & $\begin{array}{l}0.127 \\
(38)\end{array}$ & $\begin{array}{l}-0.250 \\
(20)\end{array}$ & - & $\begin{array}{l}-0.385 \\
(15)\end{array}$ \\
\hline $\begin{array}{l}\text { Age at study entry } \\
\beta_{\text {age, }} \text { year }^{-1}\end{array}$ & $\begin{array}{l}0.0649 \\
(10)\end{array}$ & $\begin{array}{l}0.0257 \\
(48)\end{array}$ & $\begin{array}{l}0.075 \\
(4)\end{array}$ & - \\
\hline $\begin{array}{l}\text { Selegiline } \\
\beta_{D P} \text { at } 10 \mathrm{mg} \mathrm{day}^{-1}\end{array}$ & $\begin{array}{l}0.925 \\
(28)\end{array}$ & $\begin{array}{l}-0.451 \\
(35)\end{array}$ & - & $\begin{array}{l}-0.466 \\
(37)\end{array}$ \\
\hline $\begin{array}{l}\text { Levodopa } \\
\beta_{L D} \text {, at } 300 \mathrm{mg} \mathrm{day}^{-1}\end{array}$ & - & - & - & \\
\hline Disease status measure & UPDRS & UPDRS & PIGD & UPDRS \\
\hline$\beta_{D S}$, per unit & $\begin{array}{l}0.0336 \\
(10)\end{array}$ & $\begin{array}{l}0.101 \\
(9)\end{array}$ & $\begin{array}{l}0.147 \\
(28)\end{array}$ & $\begin{array}{l}0.036 \\
(18)\end{array}$ \\
\hline
\end{tabular}

EOT, end of trial; UPDRS, United Parkinson's Disease Rating Scale; PIGD, postural instability gait disorder score.

The death hazard model $h$, as a function of time is:

$h$ (time) $=\beta_{0} * \exp \left(\beta_{\text {time }} *\right.$ time $+\beta_{\text {age }} * A_{g e}+\beta_{D P} * D P+\beta_{L D} * L D+\beta_{D S} *$ status (time $\left.)\right)$

Age $_{0}$ is age at study entry; DP is 1 when selegiline is used, LD is 1 when levodopa is used otherwise DP and LD are zero; Status(time) is the predicted disease status e.g. UPDRS,

at time since study entry. 


\section{Table A3}

Dropout model parameters for non-death events as a cause of dropout. RSE (in parentheses) is the relative standard error calculated as a percentage of the parameter point estimate

\begin{tabular}{|lcl|}
\hline Parameter & Estimate (RSE \%) & Units \\
\hline $\boldsymbol{\beta}_{0, \text { Gompertz, }}$ & $0.0764(22.3)$ & $1 /$ year \\
$\boldsymbol{\beta}_{1, \text { Gompertz, }}$ & $-0.417(21.9)$ & $1 /$ year \\
$\boldsymbol{\beta}_{\text {selegiline }}$ & $-1.6(13.2)$ & Selegiline used \\
$\beta_{D S}$ & $0.0391(16.9)$ & $1 /$ UPDRS \\
$\beta_{0, \text { Gompertz, }}$ & $0.0525(139)$ & $1 /$ year $\times 10^{9}$ \\
$\beta_{1, \text { Gompertz, }}$ & $3.4(5.6)$ & $1 /$ year \\
$\boldsymbol{\beta}_{0, \text { Weibull }}$ & $0.303(135)$ & $1 /$ year \\
$\boldsymbol{\beta}_{1, \text { Weibull }}$ & $2.99(27.3)$ & $1 /($ In(year) $)$ \\
\hline
\end{tabular}

UPDRS, United Parkinson's Disease Rating Scale.

The dropout hazard model $h$, as a function of time is:

$h$ (time) $=\beta_{0, \text { Gompertz, }}$ * $\exp \left(\beta_{1, \text { gompertz, } 1}\right.$ * time $+\beta_{\text {selegiline }}{ }^{*} \mathrm{DP}+\beta_{D S}$ * status $($ time $\left.)\right)$

$+\beta_{0, \text { Gompertz, }}{ }^{*} \exp \left(\beta_{1, \text { Gompertz,2 }}{ }^{*}\right.$ time $)+$ if(time $>0, \beta_{0, \text { Weibull }}$ * $\exp \left(\beta_{1, \text { Weibull }}\right.$ * In(time)), 0)

DP is 1 when selegiline is used and 0 when not. Status(time) is the predicted UPDRS score at time since study entry.

\section{REFERENCES}

1 Marras C, Rochon P, Lang AE. Predicting motor decline and disability in Parkinson disease: a systematic review. Arch Neurol 2002; 59: 1724-8.

2 Post B, Merkus MP, de Haan RJ, Speelman JD. Prognostic factors for the progression of Parkinson's disease: a systematic review. Mov Disord 2007; 22: 1839-51. quiz 988.

3 Emre M, Aarsland D, Brown R, Burn DJ, Duyckaerts C, Mizuno Y, Broe GA, Cummings J, Dickson DW, Gauthier S, Goldman J, Goetz C, Korczyn A, Lees A, Levy R, Litvan I, McKeith I, Olanow W, Poewe W, Quinn N, Sampaio C, Tolosa E, Dubois B. Clinical diagnostic criteria for dementia associated with Parkinson's disease. Mov Disord 2007; 22: 1689-707. quiz 837.

4 Lees AJ. Comparison of therapeutic effects and mortality data of levodopa and levodopa combined with selegiline in patients with early, mild Parkinson's disease. Parkinson's Disease Research Group of the United Kingdom. BMJ 1995; 311: 1602-7.

5 Olanow CW, Myllyla VV, Sotaniemi KA, Larsen JP, Palhagen S, Przuntek $\mathrm{H}$, Heinonen EH, Kilkku O, Lammintausta R, Maki-Ikola O, Rinne UK. Effect of selegiline on mortality in patients with Parkinson's disease: a meta-analysis. Neurology 1998; 51: 825-30.

6 Collett D. In: Modelling Survival Data in Medical Research, 2nd edn. Boca Raton, FL: CRC Press, 2003.

7 Lindsey JK. A study of interval censoring in parametric regression models. Lifetime Data Anal 1998; 4: 329-54.

8 Sparling YH, Younes N, Lachin JM, Bautista OM. Parametric survival models for interval-censored data with time-dependent covariates. Biostat 2006; 7: 599-614.
9 Duffull SB, Wright DFB, Winter HR. Interpreting population pharmacokinetic-pharmacodynamic analyses - a clinical viewpoint. Br J Clin Pharmacol 2011; 71: 807-14.

10 Holford NH, Nutt JG. Interpreting the results of Parkinson's disease clinical trials: time for a change. Mov Disord 2011; 26: 569-77.

11 Parkinson Study Group. Mortality in DATATOP: a multicenter trial in early Parkinson's disease. Ann Neurol 1998; 43: 318-25.

12 Marras C, McDermott MP, Rochon PA, Tanner CM, Naglie G, Rudolph A, Lang AE. Survival in Parkinson disease: thirteen-year follow-up of the DATATOP cohort. Neurology 2005; 64: 87-93.

13 DATATOP: a multicenter controlled clinical trial in early Parkinson's disease. Parkinson Study Group. Arch Neurol 1989; 46: 1052-60.

14 Jankovic J, McDermott M, Carter J, Gauthier S, Goetz C, Golbe L, Huber S, Koller W, Olanow C, Shoulson I. Variable expression of Parkinson's disease: a baseline analysis of the DATATOP cohort. The Parkinson Study Group. Neurology 1990; 40: 1529-34.

15 Fahn S, Elton R. The unified Parkinson's disease rating scale. In: Recent Developments in Parkinson's Disease, eds Fahn SMC, Calne DB, Goldstein M. Florham Park, NJ: Macmillan Healthcare Information, 1987; 153-63. 293-304.

16 Folstein MF, Folstein SE, McHugh PR. 'Mini-mental state'. A practical method for grading the cognitive state of patients for the clinician. J Psychiatr Res 1975; 12: 189-98.

17 Hamilton M. A rating scale for depression. J Neurol Neurosurg Psychiatry 1960; 23: 56-62.

18 Schrag A, Sampaio C, Counsell N, Poewe W. Minimal clinically important change on the unified Parkinson's disease rating scale. Mov Disord 2006; 21: 1200-7.

19 Schrag A, Barone P, Brown RG, Leentjens AF, McDonald WM, Starkstein S, Weintraub D, Poewe W, Rascol O, Sampaio C, Stebbins GT, Goetz CG. Depression rating scales in Parkinson's disease: critique and recommendations. Mov Disord 2007; 22: 1077-92.

20 Holford NH, Chan PL, Nutt JG, Kieburtz K, Shoulson I. Disease progression and pharmacodynamics in Parkinson disease evidence for functional protection with levodopa and other treatments. J Pharmacokinet Pharmacodyn 2006; 33: 281-311.

$21 \mathrm{Vu}$ TC, Nutt JG, Holford NHG. Progression of motor and nonmotor features of Parkinson's disease and their response to treatment. Br J Clin Pharmacol 2012; 74: 267-83.

22 Jankovic J, Kapadia AS. Functional decline in Parkinson disease. Arch Neurol 2001; 58: 1611-5.

23 Beal S, Sheiner L, Boeckmann A. NONMEM User's Guides. Ellicott City, MD: Icon Development Solutions, 1989-2009.

24 Garnett C, Holford NHG. Bone mineral density progression linked to dropout and time-to-fracture: application to postmenopausal women taking hormone replacement therapy. In: 5th International Symposium on Measurement and Kinetics of In Vivo Drug Effects, Noordwijkerhout, the Netherlands, 2006. 
25 Björnsson MA, Simonsson USH. Modelling of pain intensity and informative dropout in a dental pain model after naproxcinod, naproxen and placebo administration. Br J Clin Pharmacol 2011;71:899-906.

26 Friberg LE, de Greef R, Kerbusch T, Karlsson MO. Modeling and simulation of the time course of asenapine exposure response and dropout patterns in acute schizophrenia. Clin Pharmacol Ther 2009; 86: 84-91.

27 R Development Core Team. R: a language and environment for statistical computing. In, 2.6 Edition, Vienna, Austria: R Foundation for Statistical Computing, 2007.

28 DCCT/EDIC Research Group, de Boer IH, Sun W, Cleary PA, Lachin JM, Molitch ME, Steffes MW, Zinman B. Intensive diabetes therapy and glomerular filtration rate in type 1 diabetes. N Engl J Med 2011; 365: 2366-76.

29 Graham PL, Ryan LM, Luszcz MA. Joint modelling of survival and cognitive decline in the Australian Longitudinal Study of Ageing. J R Stat Soc Ser C Appl Stat 2011;60: 221-38.

30 Song X, Davidian M, Tsiatis AA. A semiparametric likelihood approach to joint modeling of longitudinal and time-to-event data. Biometrics 2002; 58: 742-53.

31 Deslandes E, Chevret S. Assessing surrogacy from the joint modelling of multivariate longitudinal data and survival: application to clinical trial data on chronic lymphocytic leukaemia. Stat Med 2007; 26: 5411-21.

32 Alves G, Larsen JP, Emre M, Wentzel-Larsen T, Aarsland D. Changes in motor subtype and risk for incident dementia in Parkinson's disease. Mov Disord 2006; 21: 1123-30.

33 Burn DJ, Rowan EN, Allan LM, Molloy S, O'Brien JT, McKeith IG. Motor subtype and cognitive decline in Parkinson's disease, Parkinson's disease with dementia, and dementia with Lewy bodies. J Neurol Neurosurg Psychiatry 2006; 77: 585-9.

34 Ben-Shlomo Y, Churchyard A, Head J, Hurwitz B, Overstall P, Ockelford J, Lees AJ. Investigation by Parkinson's Disease Research Group of United Kingdom into excess mortality seen with combined levodopa and selegiline treatment in patients with early, mild Parkinson's disease: further results of randomised trial and confidential inquiry. BMJ 1998; 316: 1191-6.

35 Thorogood M, Armstrong B, Nichols T, Hollowell J. Mortality in people taking selegiline: observational study. BMJ 1998; 317: 252-4.

36 Churchyard A, Mathias CJ, Boonkongchuen P, Lees AJ. Autonomic effects of selegiline: possible cardiovascular toxicity in Parkinson's disease. J Neurol Neurosurg Psychiatry 1997; 63: 228-34.

37 Turkka J, Suominen K, Tolonen U, Sotaniemi K, Myllyla VV. Selegiline diminishes cardiovascular autonomic responses in Parkinson's disease. Neurology 1997; 48: 662-7.

38 Churchyard A, Mathias CJ, Lees AJ. Selegiline-induced postural hypotension in Parkinson's disease: a longitudinal study on the effects of drug withdrawal. Mov Disord 1999; 14: $246-51$.

39 Coyle J, Hobson P, Meara J. Autonomic effects of selegiline: possible cardiovascular toxicity in Parkinson's disease. J Neurol Neurosurg Psychiatry 1998; 65: 280. author reply 81-2.

40 Tombaugh TN, Mclntyre NJ. The mini-mental state examination: a comprehensive review. J Am Geriatr Soc 1992; 40: 922-35.

41 Aarsland D, Andersen K, Larsen JP, Lolk A, Nielsen $\mathrm{H}_{\text {, }}$ Kragh-Sorensen P. Risk of dementia in Parkinson's disease: a community-based, prospective study. Neurology 2001; 56: 730-6.

42 Marsh L, McDonald WM, Cummings J, Ravina B. Provisional diagnostic criteria for depression in Parkinson's disease: report of an NINDS/NIMH Work Group. Mov Disord 2006; 21: 148-58.

43 Vu TC, Nutt JG, Holford NH. Time course of disease status as a predictor of clinical outcome in Parkinson's Disease. Available at http://www.page-meeting.org/?abstract=1256 (last accessed December 2010). In: PAGE, 2008: Abstr 1256. 